\title{
Cultural diversity: A qualitative study on Saudi Arabian women's experience and perception of maternal health services
}

\author{
N. Karout ${ }^{1}$, S.H. Abdelaziz ${ }^{1}$, M. Goda ${ }^{1}$, S. AlTuwaijri ${ }^{2}$, N. Almostafa ${ }^{3}$, R. Ashour ${ }^{3}$, H. Alradi ${ }^{3}$ \\ 1. Saad Nursing College and Allied sciences, Nursing Department, Saudi Arabia. 2. Clinical research laboratory, Saad \\ research and developing center, Saad Specialist Hospital, Saudi Arabia. 3. Saad Specialist Hospital, Saudi Arabia
}

Correspondence: Najwa Kamel Karout. Address: Saad nursing college, Saudi Arabia. Email: Nkkarout@yahoo.com.

Received: January 30, 2013

DOI : 10.5430/jnep.v3n11p172
Online Published: July 24, 2013

URL: http://dx.doi.org/10.5430/jnep.v3n11p172

\section{Abstract}

Background: The Saudi Arabian healthcare system is mainly staffed by Non-Saudi health professionals who are recruited from all over the world. At times, inadequate cultural awareness by health professionals can render their caring for Saudi patients inadequate and thus more challenging for the women. When nurses and patients belong to different cultures, culture-based misunderstandings can influence their relationships and interactions adversely. The aim is to describe the experiences of women who were admitted to maternity wards gaining their perceptions and understanding of the cultural diversity exhibited by health care providers.

Methods: A qualitative phenomenological approach was utilized for the exploration of the experiences and perceptions of 37 Saudi women who admitted to a private hospital in Saudi Arabia and related to cultural diversity of health providers' team. The principal method of data collection was a semi structured, open-ended interview. Focus of enquiry was the experience of the participants during their stay in maternity wards and then depending upon the answers further discussion was subdivided into questions about gender, believes and values, religion, language and the nationality of the health providers.

Findings: The findings were grouped into five themes, namely: religious diversity, linguistic diversity, gender diversity, values and beliefs diversity and nationality diversity. The themes have been discussed as representing key experiences and perceptions that can positively and negatively affect maternity services provided by nurses.

Conclusion: Understanding the concept of culture and the factors related to it will enhance the quality of care provided by the health team and may also play a positive role during recruitment of the health team providers and in the provision of any continuous education programmes.

\section{Key words}

Culture diversity, Maternity care, Saudi women perception, Saudi women experience

\section{I ntroduction}

An emerging global reality is the presence of a culturally diverse workforce in international health care environments. Not only are nurses delivering care to increasingly culturally diverse clients, the nurses themselves may come from different 
cultural and educational backgrounds. In order to provide culturally competent health care, such diversity must be molded into professional collaboration and respect ${ }^{[1]}$.

The United Arab Emirates (UAE) has become a linguistically and culturally diverse society where the majority of health care's staff drawn from a range of non-Arabic speaking backgrounds. In hospitals, the resultant mix of language and cultural differences highlights the importance of the role of nurses in facilitating the quality of care as the primary healthcare workers communicating and interacting with both patients and colleagues, therefore in the Arab countries the presence of those health providers with their different believes and cultures needs to be evaluated and assessed ${ }^{[2]}$.

The Saudi Arabian health system is mainly staffed by Non-Saudi health professionals recruited from all over the world. At times, inadequate cultural awareness by health professionals can render their caring for Saudi patients problematic. It is possible, however, that this problem can be addressed should aspect of Saudi culture be introduced to health professionals intending to care for in Saudi patients ${ }^{[3]}$.

The practice of midwifery is a reflection of the spectrum of diversity among midwives and the women they serve. However the composition of the midwifery workforce does not reflect the diversity of the women for whom they provide care. Therefore providing culturally appropriate care for women requires our best understanding of their believes, needs, and desires ${ }^{[4]}$.

\subsection{Cultural diversity}

Cultural diversity is a type of culture with the following specific characteristics: co-existence of different groups, e.g. ethnic, religious, linguistic and other groups each with their own values and belief systems, traditions and different lifestyles ${ }^{[5]}$. People belonging to different cultures may have different kinds of demands in terms of health. It is an essential human right for everyone to express freely their own cultural values. People having different cultural values should be respected in terms of their cultural values and the health care they are to be given should be offered considering this fact ${ }^{[6]}$.

Providing care to culturally diverse population in which a challenge for nurses. So nurses need to recognize the concept of transcultural nursing and to be competent to deliver the comprehensive care. To be culturally competent the nurse needs to understand her own world views and those of the patient, to avoid stereotyping and misapplication of scientific knowledge. Cultural competence includes obtaining cultural information and then applying that knowledge. Knowledge of cultural information is very essential for delivery the quality of care and it improves health outcomes ${ }^{[7]}$. Cultural diversity is a challenge for community nurses and can present many difficulties in the provision of quality nursing care and in achieving optimal health outcomes ${ }^{[8]}$. Culture defines how persons behave towards each other. When nurses and patients belong to different cultures, culture-based misunderstandings could influence the nurse-patient relationships and interacttions adversely ${ }^{[9]}$.

\subsection{The concept of culture and nursing}

The concept of culture has been explained in different theories in sociology, psychology and nursing theory. The previous studies showed the importance of the culture in clinical experience and nursing practice, the theory initiates from clinical experiences recognizing that culture, a holistic concept, was the missing link in nursing knowledge and practice. Through a creative process of concept explication, reformulation, and re-synthesis, the theory of cultural care was set forth as a guide for the development of nursing knowledge ${ }^{[10]}$. The concept of culture was derived from anthropology and the concept of care was derived from nursing. The theorist holds that cultural care provides the broadest and most important means to study, explain, and predict nursing knowledge and concomitant nursing care practice. The ultimate goal of the theory is to provide cultural congruent nursing care practices ${ }^{[10]}$. Transcultural nursing is a major component of a professional nurse practice model which provides a visionary perspective for nursing care. Within the practice model, transcultural care principles are used to guide education, clinical practice and nursing research. Furthermore, to identify ethical issues faced by the nurses in the transcultural setting ${ }^{[1]}$. Leininger's theory of Culture Care Diversity and 
Universality has made a major contribution to the discipline of nursing. With care and culture as central to the theory, the ultimate goal of the theory is to improve client care ${ }^{[11]}$.

Previous transcultural nursing studies of birthing in different cultures with a care focus was held as essential to understand health care practices and wellbeing. The need exists for further in depth studies of care meanings and practices for women of diverse cultures will significantly encourage holistic and humanistic care ${ }^{[12]}$.

\subsection{Nursing in a Muslim culture}

An ethnographic and ethno nursing study has been conducted in US urban community to describe and analyze the meanings and experiences of care for Lebanese Muslims as influenced by cultural context in selected natural and health care settings, Universal themes of care that were similar in the three contexts reflected care as a religious obligation in Islam, care as equal but different gender role responsibilities, and care as individual and collective meanings of honor. Major diversities in the hospital context reflected areas of cultural incongruence between Lebanese Muslim clients and health personnel with respect to gender role differences and certain American models of care practices, therapies and ethical codes which did not fit the Lebanese Muslim cultural life ways, values and beliefs. Diversity in the clinic context reflected incongruence regarding expectations of responsible prenatal care. Other findings of the study related to professional and folk beliefs and practices, semantic descriptors of care in the Arabic language, and gender differences in perceptions of a caring and non-caring nurse ${ }^{[6]}$.

Other research results indicated that non-Muslim nurses lacked knowledge about Muslim practices concerning breastfeeding, Ko'hl, the "evil eye", modesty, and medicine and food taboos. If these aspects could be addressed during the recruitment and in-service education of non-Muslim nurses working in Muslim countries, this could enhance the quality of culture-competent nursing care ${ }^{[9]}$.

Global nursing shortages have necessitated closer scrutiny of recruitment and retention initiatives of nurses both locally and internationally. For many decades the nursing workforce of Saudi Arabia relied on international expatriates to be the backbone of the industry. In recent years however, Saudi women have been recruited into nursing preparation courses conducted at degree level ${ }^{[13]}$, but as the number of Saudi nurses recruited is not enough to cover the needs of the health settings, the majority of the health system in Saudi still in need to recruit non-Saudi nurses especially those who will provide care in maternity health settings. Previous study showed that nurses need to be culturally competent in the prenatal care of women from Saudi Arabia. A basic understanding of the Arab culture, attitudes, values, beliefs and practices is

addressed ${ }^{[14]}$. As it is very important to understand the Saudi women's believes, values and perception related to the maternity care provided by diverse nurse.

The purpose of this study was to describe the experiences of women who were admitted to maternity ward of a private hospital in Al-Khobar city and to explore their perceptions of the level of cultural diversity they experienced care provided. It is hoped that studying their experience and perception will further assist in creating a better understanding of the concept of culture in healthcare and the factors that are important in enhancing the quality of care provided by the health team. Furthermore these results can also be considered as assisting in tailoring both the recruitment of the health team members and in devising appropriate continuous education programmes that prepare expatriate nursing practitioners for work in Saudi Arabia.

\section{Methods}

\subsection{Study design}

A qualitative phenomenological approach was utilized in the exploration of the experiences and perception of Saudi women related to cultural diversity of the health providers' team admitted to a private hospital Al-Khobar city and accepted to participate in the study. A phenomenological approach was used in order to obtain clear descriptions of the lived 
experience of individual women from their perspective, focusing on the primacy of her subjective experience ${ }^{[15]}$. It is deemed to be an appropriate method in order to identify major and minor themes expressed by the participants ${ }^{[16]}$. Cultural care diversity served as the conceptualizing framework for the study. It was predicted that care meanings for the health care provider are largely influenced by and derived from cultural context through shared cultural values, beliefs and practices. A previous study showed that nurses, with cultural diversity, bring with them cultural beliefs, attitudes, language expressions and communication patterns which are meaningful to them when they enter a health care setting ${ }^{[17]}$.

\subsection{Data collection}

In this phenomenological qualitative study the method of data collection was a semi structured, open-ended interview format that resulted in obtaining bright descriptions of the experience of women. A purposive sample consisted of 37 women admitted to the maternity wards during the period of October 2012. In a phenomenological study, a sample size of 10-15 participants is acceptable and valid ${ }^{[18]}$. The primary question was focused on the participants' experience during their admission in the hospital at the different maternity wards and then according to the answers generated by interviews further discussion was conducted under sub questions about gender, believes and values, religion, language and the nationality of the health providers.

\subsection{Trustworthiness}

The validity of the questions was obtained from previous studies where themes related to culture diversity model and transcultural nursing model had emerged ${ }^{[6,10]}$. Semi-structured interviews, were conducted to clarify, expand, and confirm the emerging themes. Peer reviewed journals provided a further source of data for the researchers and assisted in the development of personal reflections and methodological decisions. This also ensured trustworthiness of the data. Trustworthiness was further assured by conducting a 45 minutes to one hour interviews, concurrent data collection and analysis, verbatim transcription and member checks was undertaken ${ }^{[19]}$. To assure the validity of the interviews, the interview was conducted with 2 groups of trained interviewers. Each group included 2 persons, one conduced the interview and the other was the observer, who took notes and recorded the interview, in order to assure that the questions were understood and discussed freely. The researcher stopped the interviews when the themes appeared relevant and flexible enough to catch the individual experiences, and the data remained unchanged. The interviewers were Saudi new graduate nurses. This was because it was assumed that in line with previous studies, as the patients were mostly Saudi, they would share their experiences more openly with peers than with international researchers ${ }^{[20,21]}$.

\subsection{Ethical considerations}

Institutional Review Board approval was granted by the hospital in which the researcher was enrolled while completing the study. Individual participation in the study was voluntary. The consent form was reviewed with the participants prior to the start of the interview and signed. Participants received assurance of confidentiality and anonymity as the use of a coding system for all participants' names ensured that confidentiality was maintained.

\subsection{Data analysis}

The data were analyzed by inductive content phenomenological analysis that consists of several steps where the researcher has to become immersed in the data and significant statements are identified. Data collection and analysis were completed simultaneously ${ }^{[22]}$. Five themes were recognized discussed as experiences affect the maternity services provided by nurses: (i) linguistic diversity, (ii) religion diversity, (iii) gender diversity, (iv) values and believes diversity, and (v) nationality diversity.

\section{Findings}

The participants were Saudi, their ages ranged from 21-35 years old, the time of admission was from two to five days, and the majorities were multi-Para and had a previous admission experience in the maternity ward of the hospital. 


\section{Theme 1:}

(i) Linguistic diversity: The biggest problem that was discussed by most of the participants was that of linguistic diversity. This was understood as not only the difference between English [spoken by all of the nurses] and Arabic language [spoken by most of the women] and extended to the English accent by non-English speakers (Indians, Filipino, Pakistanis,). Allied to this the Arabic speaker's accent emanating from different Arabic countries (Egyptian, Lebanese, Moroccan and other countries in the Middle East) was highlighted as a problem facing the participants. In addition it was explained by some participants that aspects of nonverbal communication was also not understandable such as forms of signs that were used and some facial expressions.

...I thought I know English at the level that I could understand the advice given by the Indian midwife when she was telling me what to do while she assess the contraction, she repeated twice something that I did not understand ,I asked her to repeat maybe I could discover the accent she speaks, but finally she asked another nurse to translate for me, I felt embarrassed.

...It was hard to remember when suddenly a quick movement has been happened, looking to the face of the midwife as she was very worried asking help from other person, discussing something about me and asking me to do something or to answer as I did not know because they were discuss in their language and accent .

...When I came for prenatal care the Filipino nurse started asking me in her Arabic broken language some questions that most of the time I could not understand, then when I went to be checked by the Egyptian physician, she asked me in Arabic "why did not you come when you saw amniotic fluid" based on the nurse report as she thought I have ruptured membrane. Some information also was misunderstood because of the questions in Arabic accent of the Filipino nurse.

...I had hypertension and I was admitted before labor for one week. My baby birth was too difficult and also my baby had complication, the nurses were informing me about everything that was happening, always I needed to wait for Arabic speaker or translator to report to me what should I do because I could not understand the Arabic language of the Indian or their English accent.

\section{Theme 2:}

(ii) Religion diversity: This was considered by the women as one of the issues that have been experienced when receiving health services at the hospital maternity wards. The women expressed that religion and religious beliefs are important factors that assist in making them feel comfortable, especially in the examination room, and during labor. According to them, in such places, the presence of non-Muslim service providers did not satisfy them because they believed that Muslim service providers will not look at their uncovered bodies especially their genatalia unless there is a need for that, and they will be conscious uncovering themselves.

...I've been very embarrassed, and confused when the midwife nurse asked me to remove my underwear and open my legs while there was a possibility for some staff to pass by. If she was a Muslim, maybe she would keep the importance of this issue in her mind.

....I've experienced some fear and shyness when the staffs were preparing me for the operation, I was requested to be totally naked. If the nurses and physicians were Muslims, they would deal with unclothed women in a different manner.

....Being a Muslim lady surrounded by non-Muslim nurses or physicians made me feel confused, it was an annoyed experience when I was requested to be examined by the physician and the nurse who has been 
unnecessarily looking at my private and banned places, even I had my veil "Nikab". From my point of view, if the nurse had Islamic believes, she would protect my privacy more.

...As a part of her job, the nurse used to unconsciously open my door at any time, causing interruption of me especially during my praying. Moreover, touching all my properties including the holy Quran while she is a nonMuslim, that thing absolutely annoyed me.

\section{Theme 3}

(iii) Values and believes diversities: Most of the participants have their own believes related to pregnancy, labor and birth. There are many traditions and values associated with believes which were mentioned during the interviews and did not violate many of the studies that have been conducted to assess the effect of traditions and values on health practices to citizens. But here, the experience of women with health providers was unexpected because of the existed different traditions and values between them and the staffs, the value of pronouncing the name of God at the starting of any procedure to kept from harm such as breastfeeding, assessment of the abdomen, vaginal examination, giving birth, this value is strongly affected by the participants' believes that they will be hurt, or their baby will face a harm, or their breastfeeding will failed.

...... Recently I have given birth my first baby, but I do not know why the nurse said "big baby, big baby" and every nurse when they saw my baby they shouted "big baby" without mentioning the name of God "Machallah" or "Bismillah" that would badly affect my baby's health. So it was a bad experience that annoyed me and made me feel angry and unsatisfied.

..... I was upset when the physician put her hand on my abdomen to assess the movement of my embryo and said that my baby move too much, I felt that my baby will hurt because of her evil eye.

.... I have been very concerned when I was breastfeed my baby and the nurse was helping me to hold him, I had pain because of the cesarean operation but the nurse was looking to my breast, I felt the milk producing will stop because of her wondering on the quantity of milk produced for the first letdown .

.... I like pronouncing the name of God when dealing with me, like giving birth or breastfeeding and also when dealing with my baby or even assessing and touching him, as I think this will keep him save from any harm. Unfortunately this did not happen with non-Saudi nurses.

\section{Theme 4}

(iv) Gender diversity:

This theme was among the subjects that have been reported by the participants, some participants had negative experience when treated by a male providing health services in the labor room or during vaginal examination and normal delivery while other participants insisted on the presence of a male during surgery as positive experience.

... I felt more comfortable when I saw the staffs around me were all females. I think being dealt by females in maternity wards is better than males because of the private situation there...Despite my request of being treated by females and only females I wondered that one of the staff was male, I felt anger but I was too weak to scream get out...they should respect my desire.

... I found some complications during birth that it was indicated to move quickly and take an important decision to follow and handle my case, I thank God because there was a male physician, I think male better than female during handling any complication especially when it needs surgery. 
... I do not care if the nurse or physician was male or female in operation room and after being sleep, but during normal labor female is better because she will feel and deal better with us ,so just I was worried to be obliged dealing with male in labor room .

\section{Theme 5:}

(v) Nationality diversity: The participants' perception about national diversity was identified as being important.

The participants had preferences on non-Saudi nurses especially Filipino and Indian. They perceived the nurse as a maid or house keeper. From their point of view, the role of nurses is changing blankets, help them to enter the toilet, change bloody under wear, also give them the slippers, clean their perineum and give the participant her mobile phone from the neighboring table. According to them, the above mentioned services are attainable only by nationalities such as Filipino and Indian.

...During my days of admission in maternity wards I could not ask the nurse all the services I need, the Filipino or Indian accept to do anything I ask but not the Jordanian or Lebanese or any other Arab nationality maybe in my point of view we are used to deal with these nationalities as housekeepers.

...The Saudi nurses usually know us directly or indirectly and being in maternity wards have some privacy so during my sitting in the hospital I was worried from the Saudi nurses that may had curiosity about my private situation and will know everything about me that I do not like.

...During my sitting in maternity ward I was asking the nurse to provide me the slippers or to help in washing my face or changing my pads. The non-Arab nurses' nationality was more cooperative than the Arab nationality nurses, who were proud but the non-Arab were modest and helpful.

\section{Discussion}

As previous studies have shown that the nation's hospitals face increasing demands to participate in a wide range of quality improvement activities, the role and influence of nurses in these efforts is also increasing, because they are the key caregivers in hospitals. They can significantly influence the quality of care provided and the patient outcomes achieved ${ }^{[23]}$. When nurses and patients belong to different cultures, culture-based misunderstandings could influence their relationships and interactions adversely ${ }^{[24]}$.

Participants considered that there were many factors affecting their experience. It is not surprising that one of these factors was the linguistic diversity among staff in the maternity wards, which was consistent with many other studies that identified the importance of the linguistic diversity among staff ${ }^{[17]}$.

It is obvious that the difference in language even in accent is considered as a barrier in communication. This barrier leads to misunderstanding of the messages given by the nurses to the women such as patient education and counseling. Providing care in the maternity ward requires an implementation of different procedures that need complete corporation from the women and adherence to instructions that will be given to them such barrier revealed in the finding of this study an important issue that was considered by the women as a negative experience and potentially life threatening, such situation could be prevented by conducting a cultural assessment which accommodated the patient's cultural values into the care plan ${ }^{[25]}$.

Maternity services struggle to provide culturally appropriate care that meets the needs of women from diverse populations. Problems include simplistic understandings of ethnicity and the role of culture in women's lives. 
In this study, participants recognized that there were some religious and gender based issues that affect the quality of health services provided. This was confirmed by the previous studies which showed that the religious believes are very important in patient's culture and health practices .Some people wear amulets to be protected from evil eyes or they burn incense to keep it away from the sick people. Such practices are declining and are used primarily to avoid illness and harm to a healthy person ${ }^{[14]}$.

Lack of knowledge among nurses concerning the religious and cultural practices has found in a previous study from the perspective of immigrant Muslim women. In addition this lack of knowledge also acted as barrier for women seeking access to maternal health services. Health information was limited or lacked the cultural and religious specificity to meet their needs during pregnancy, labor and delivery, and postpartum phases. There were also significant gaps between existing maternity health services and women's needs for emotional support, and culturally and linguistically appropriate information, as recommendation for change directed at decision makers include improving access to culturally and linguistically appropriate maternity and health related information, developing the diversity responsiveness of health care providers ${ }^{[26]}$.

In this study some of the participants insisted on the evil eye of Non-Muslim nurses or physicians that may hurt them or their babies while looking during giving birth, breast feeding, or touching their abdomen to assess foetal movement. This presents a dilemma due to the belief in the concept of potential harm from the 'evil eye' and the necessity for various health professionals to engage in assessment activity necessary to plan and deliver necessary care such as instructing the mother about assessing foetal movement or the most successful way of breastfeeding, all of which can be misunderstood if not conducted with understanding and sensitivity. Such issues can be simply prevented by increasing awareness among healthcare providers about relevant beliefs and Islamic religious practice issues as understood and practiced in Saudi Arabia. Adherence to such particular set of believes, values, and practices that constitute the patients' religion should be of considerable importance to healthcare professionals because they are of great importance to women and their families. It is therefore essential that nurses should acquire at least a basic knowledge of the most common religions in order to deliver holistic care that meets their patients' needs ${ }^{[27]}$.

Muslim women and their perception about providing care by opposite gender were assessed and reflected important issue and solutions to prevent any related problem ${ }^{[28]}$. Based on religious and cultural issues, the results of this study showed that there was a preference among Saudi women to be with a female rather than a male during birth or during any gynecologic assessment. For pregnancy or gynecological needs, women prefer a female doctor. However, in Arab culture, women are reluctant to discuss these concerns with men. Sexual problems and venereal diseases are difficult for Arab patients to deal with. Women are shy to talk about their private lives and may feel more comfortable with women doctors ${ }^{[14]}$.

Despite the desire of the participants to deal with female nurses, they are not reluctant to be treated by male surgeons when they need an emergency cesarean. It shows gender inequality in the profession, as they look to females as a helper and to males as physicians. This concept has been also addressed in previous studies ${ }^{[29,30]}$.

Within Saudi Arabia it could be argued that the nature of nurses' work, especially Saudi nurses, in hospitals is not well understood by the public or indeed by policymakers. In a recent survey, results showed that the public is confused about what nurses do and what distinguishes them from nurse aides and other less trained personnel. The public understands that nurses' work is physically and emotionally demanding but may view this work as delivering care as ordered and providing physical and emotional comfort to patients and their families ${ }^{[31]}$.

Cultural stereotyping is one of those problems showed in a study conducted on women of Bangladeshi origin where cultural context made a difference to their experiences of breast-feeding and breast-feeding support. Some practitioners used cultural stereotypes which, combined with organizational constraints, resulted in services not meeting many of the women's needs ${ }^{[32]}$. During working with the Saudi nurses, the researcher have recognized this stereotyping issue among 
the students themselves "as future patients" perceive the Indian nurses as housekeepers and they do not accept them as preceptors even though they are nursing students and may face the same problem with the patients. This perception is a social phenomenon and needs to be addressed by social scientists, especially since the participants were insisting on considering black skinned women from Sri Lanka, India, Bangladesh or Ethiopia as house maids, because they used to bring housekeepers from those countries and their faces become a symbol of housekeepers not any other profession. However, previous studies conducted on the impact of color on the provision of services in nursing showed that there is some discrimination still existed in the perception of the patients regarding the skin color of the nurse ${ }^{[17]}$.

\section{Conclusion}

The aim of this study was to describe the lived experience of Saudi women in the maternity ward at a hospital where nursing care was provided by staff group with different cultural background. It was concluded that participants' perceived experience was related to many essential components principally barriers related to their culture, beliefs and values.

To optimize the role of nurses in quality improvement, it is important that continuous nursing education programmes strengthen curricula to emphasize the concepts and skills needed to participate in quality improvement activities in a host country ${ }^{[23]}$. It is suggested that there is a clear need to prepare expatriate nurses to be more adapted with the Saudi expressions and terminology related to women's health. In order to increase the quality of maternity care provided by non - Arab nurses also it is recommended that instead recruiting translator, ask the new employees to pass an Arabic Exam (Test of Foreign Arabic Language TOFAL) as a condition for employment. It is also recommended that human resources departments consider adding the passing of an Arabic proficiency exam as criteria for renewing the contracts of employees.

It is recommended that a health education programme to be developed by national ministries of health or by hospitals including counseling sessions in order to discuss with the women their religious attitude and believes accordingly and in providing educational sessions about women's health values and misconceptions. These sessions should be supported by the existence of multilingual media including leaflets and web-based resources addressing issues of central importance for care practice.

Arranging a visit by a Muslim health staff, preferably of the same sex and ethnic background, may lead to better understanding of these difficulties. Privacy is very important, especially in the presence of mixed gender health-care professionals. Accordingly it is advised that whenever health-care workers of the opposite gender enter the patient's room, a warning should be given so that the individuals may need to arrange their attire appropriately, especially for Muslim women who cover their hair.

This study has provided the ability to pause and reflect regarding the participants' thoughts about the nursing profession. Perceiving the nurse female and her role according to her nationality and asking her to provide some services during hospital admission as a "housekeeper" is an important point and prohibits the ability of the nurse to provide the care that she has been educationally and clinically prepared to provide, thus disadvantaging the patient who may initially be resistant to interventions from nurses. Maternity services struggle to provide culturally appropriate care that meets the needs of women from diverse populations and the fundamental problems would appear to stem from issues such as simplistic understandings and misunderstandings of ethnicity and the role of culture in women's lives.

The phenomenon of stereotyping should be highlighted, and discuss in the nurses committees, the nurses organizations, nursing conferences and with the decisions makers in ministries of health and education, so that activities can be developed to address the role of nurses and the nursing profession in general amongst societal groups including activities aimed at highlighting the role of nurse in mass media. It also needs to be worked on to introduce this type of education in 
universities and private institutes teaching nursing to change these perceptions among Saudi nurses who will work in the future with a multi-cultures team, as they will acquire knowledge and experience from them ${ }^{[33]}$.

It is true that the nurse's role is to provide services and care and may include also the services mentioned by the participants, but providing such services should not be under the mentioned concept "housekeepers". If nurses are to be perceived as helpers, and not health care professionals then their suggestions and advice will not be taken seriously. So it is suggested that nurse managers may need to undertake sustained efforts to explain the nurse's role to the patients ${ }^{[14]}$. However, health care providers must realize that addressing cultural diversity beyond knowing the values, believes, practices and customs of the people in addition to racial classification and national origin and that there are many other faces of cultural diversity. Religious affiliation, language, physical size, gender, sexual orientation, age, disability (both physical and mental), political orientation, socio-economic status, occupational status and geographical location are but a few of the faces of diversity ${ }^{[34]}$. So implications for education, policy and practice include the need for training of health practitioners to work with diverse populations, implementing evidence-based practice and providing an organizational context which supports practitioners to respond to diversity without using cultural stereotypes is a must ${ }^{[32]}$. Finally, cultural generalizations will not fit easily in the provision of care for every patient, but awareness of broad patterns can give practitioners a starting point from which to provide appropriate care ${ }^{[35]}$.

\section{Acknowledgment}

A special thank for Dr. Mona Goda and Mr. Jerome Marley for their assistance in reviewing and editing the paper.

\section{References}

[1] Tortumluoglu G, The implications of transcultural nursing models in the provision of culturally competent care. ICUs \& Nursing Web Journal (ICUS) issue 25, 2006.

[2] El-Amouri S, OÂ Neill S. Supporting cross-cultural communication and culturally competent care in the linguistically and culturally diverse hospital settings of UAE, Contemporary Nurse: A Journal for the Australian Nursing Profession. 2011; 39(2): 240-55.

[3] Al-Shahri MZ. Culturally sensitive caring for Saudi patients. Journal of Transcultural Nursing. 2002; 13(2): 133-8. PMid:11951716 http://dx.doi.org/10.1177/104365960201300206

[4] Kennedy HP, Erickson-Owens D, Davis JAP. Voices of diversity in midwifery: a qualitative research study. Journal of Midwifery \& Women's Health. 2006; 51(2): 85-90. PMid:16504904 http://dx.doi.org/10.1016/j.jmwh.2005.07.007

[5] Geyer N, Peu MD, Roussouw S, Morudi J, Uys E. Contributing to the ICNP: validating the term cultural diversity. Curationis. 2005; 28(2): 61-9. PMid:16045112 http://dx.doi.org/10.4102/curationis.v28i2.952

[6] Luna LJ. Care and cultural context of Lebanese Muslims in an urban U.S. community: an ethnographic and ethno nursing study conceptualized within Leininger's theory. PhD thesis. Wayne State University. 1989.

[7] Ansuya A. Transcultural nursing: cultural competence in nurses. International Journal of Nursing Education. 2012 ; $4(1)$ : 5-7.

[8] Omeri A, Malcolm PC .Cultural diversity: a challenge for community nurses Contemporary Nurse: A Journal for the Australian Nursing Profession. 2004 Oct; 17(3): 183-91.

[9] Sidumo, EM, Ehlers, VJ, Hattingh, SP. Cultural knowledge of non-Muslim nurses working in Saudi Arabian obstetric units. 2010 Sep; 33(3): 48-55.

[10] Leininger MM. Leininger's theory of nursing: cultural care diversity and universality. Nursing Science Quarterly (NURS SCI Q). 1988; 1(4): 152-60. PMid:3205480 http://dx.doi.org/10.1177/089431848800100408

[11] Rosenbaum JN. Leininger's theory of Culture Care Diversity and Universality: transcultural critique. Journal of Multicultural Nursing \& Health. 1997; 3(3): 24-9, 36.

[12] Lamp JK, Generic and professional culture care meanings and practices of Finnish women in birth within Leininger's theory of culture care diversity and universality. PhD thesis. Wayne State University, 1998

[13] Miller-Rosser K , Chapman Y ,Francis K. Historical, cultural, and contemporary influences on the status of women in nursing in Saudi Arabia. Online Journal of Issues in Nursing. 2006; 11(3): 1.

[14] The Middle Eastern Community - Transcultural Nursing. Available from: www.culturediversity.org/mide.htm (12 January 2013 last access) 
[15] Lapidus Graham J. The Lived Experience of Participation in Student Nursing Associations and Leader ship Behaviors. A Phenomenological Study, Journal of the New York State Nurses Association. 2012; 43(1): 4-12. PMid:23189510

[16] Patten M. Understanding research methods: An overview of the essentials (6thed.). Clendale. California: Pyrczak Publishing. 2007.

[17] Spangler ZDL, Nursing care values and care giving practices of Anglo-American and Philippine-American nurses conceptualized within Leininger's theory. PH.D thesis. Wayne State University, 1991.

[18] Moustakas C. Phenomenological Research Methods. Thousand Oaks. California: Sage Publishing. Inc 1994.

[19] Chapman R, Orb A. The Nursing Students' Lived Experience of Clinical Practice. The Australian electronic Journal of Nursing Education. 2000; 5(2).

[20] Rew L, Bechtel D, Sapp A. Self-as instrument in qualitative research. Nursing Research. 1993; 42(5): 300-301. PMid:8415047 http://dx.doi.org/10.1097/00006199-199309000-00012

[21] Miller J, Glassner B. The “inside” and the “outside”. Finding Realities in Interviews. In: Silverman D. (Ed.), Qualitative Research. Theory, Method and Practice. Sage Publications, London. 2004. p. 125-139.

[22] Omery A. Phenomenology: a method for nursing research. Advances in Nursing Research.

[23] Debra A., Laurie E, Felland A, Liebhaber LM. The Role of Nurses in Hospital Quality Improvement, HSC Research Brief No. 3 March 2008.

[24] Sidumo EM, Ehlers VJ, Hattingh SP. Cultural knowledge of non-Muslim nurses working in Saudi Arabian obstetric units. 2010 Sep; 33(3): 48-55.

[25] Jeffreys M. Dynamic of Diversity, Becoming Better Nurses through Diversity Awareness, 2008. Available from: www.nsna.org.

[26] Reitmanova S, Gustafson DL. "They can't understand it": maternity health and care needs of immigrant Muslim women in St. John's, Newfoundland. Maternal and Child Health J. 2008; 12(1): 101-11. PMid:17592762 http://dx.doi.org/10.1007/s10995-007-0213-4

[27] A Dictionary of Nursing, Religion and nursing practice, 2008

[28] Alberta health services, Health Care and Religious Believes. 2009. P. 22.

[29] Ulrich B. Gender Diversity and Nurse-Physician Relationships, virtual Mentor. 2010 January; 12(1): 41-45.

[30] Balayla J. Male physicians treating female patients: issue, controversies and gynecology. Mcgill J Med. $2011 ; 13$ (1): 72.

[31] Needleman J, Hassmiller S. The Role Of Nurses In Improving Hospital Quality And Efficiency: Real-World Results. Health Affairs. 2009; (28): 4.

[32] McFadden A, Renfrew MJ, AtkinK. Health Expectations: an International Journal of Public Participation in Health Care and Health Policy. 2012.

[33] Luna L. Culturally competent health care: a challenge for nurses in Saudi Arabia, Journal of Transcultural Nursing, 1998 Jan-Jun; 9(2): 8-14. PMid:9856009 http://dx.doi.org/10.1177/104365969800900203

[34] Campinha-Bacote, J. "Many Faces: Addressing Diversity in Health Care". Online Journal of Issues in Nursing. $2003 ;$ 8(1): 1. PMid:12729453

[35] Galanti G A. Iranian births. West J Med. 2000 July; 173(1): 67-68. PMid:10903305 http://dx.doi.org/10.1136/ewjm.173.1.67 